\title{
Structural Estimation of the Effect of Out-of-Stocks
}

\author{
Andrés Musalem \\ Fuqua School of Business, Duke University, Durham, North Carolina 27708, amusalem@duke.edu \\ Marcelo Olivares \\ Decision, Risk and Operations Division, Columbia Business School, Columbia University, \\ New York, New York 10027, molivares@columbia.edu \\ Eric T. Bradlow, Christian Terwiesch \\ The Wharton School, University of Pennsylvania, Philadelphia, Pennsylvania 19104 \\ \{ebradlow@wharton.upenn.edu, terwiesch@wharton.upenn.edu\} \\ Daniel Corsten \\ Operations and Technology Management, IE Business School, 28006 Madrid, Spain, \\ daniel.corsten@ie.edu
}

\begin{abstract}
$\mathrm{W}$ e develop a structural demand model that endogenously captures the effect of out-of-stocks on customer choice by simulating a time-varying set of available alternatives. Our estimation method uses store-level data on sales and partial information on product availability. Our model allows for flexible substitution patterns, which are based on utility maximization principles and can accommodate categorical and continuous product characteristics. The methodology can be applied to data from multiple markets and in categories with a relatively large number of alternatives, slow-moving products, and frequent out-of-stocks (unlike many existing approaches). In addition, we illustrate how the model can be used to assist the decisions of a store manager in two ways. First, we show how to quantify the lost sales induced by out-of-stock products. Second, we provide insights on the financial consequences of out-of-stocks and suggest price promotion policies that can be used to help mitigate their negative economic impact, which run counter to simple commonly used heuristics.

Key words: aggregate demand estimation; Bayesian methods; choice models; data augmentation; inventory management; out-of-stocks; retailing

History: Received August 6, 2008; accepted February 12, 2010, by Wallace Hopp, marketing. Published online in Articles in Advance May 17, 2010.
\end{abstract}

\section{Introduction}

In retailing, inventory decisions have direct implications on product availability to customers. When making decisions related to the inventory levels of a product category, store managers need to balance the costs of holding and replenishing inventory versus the costs of out-of-stocks. The cost of holding inventory can be calculated directly using financial measures available to store managers. In contrast, evaluating the cost of an out-of-stock requires estimating its impact on customers' buying behavior. The lack of precise measures of the costs of out-of-stocks has been cited as one of the root causes for the slow adoption of quantitative models in inventory management by practitioners (Zipkin 2000).

In terms of the magnitude and prevalence of this problem, out-of-stocks are certainly not uncommon in retailing. The average out-of-stock rate in the United States and Europe is about $8 \%$, and the costs associated with out-of-stocks vary across product categories and can be substantial in some cases. ${ }^{1}$ To quantify the financial consequences of out-of-stocks, it is useful to analyze the choices that a customer facing an out-ofstock could make. First, a customer encountering an out-of-stock may choose to defer its purchase until the desired focal product becomes available. Second, the customer may choose to purchase a substitute product in the category. Third, a customer may decide not to purchase any products-that is, the out-of-stock leads to a lost sale-which has the largest negative shortterm financial impact for the retailer. Our main objective is to develop a methodology to quantify the effect of the two latter scenarios, product substitution and lost sales, that can be implemented using information commonly available to a store manager.

A major challenge in estimating the impact of outof-stocks on retail demand is the lack of precise

\footnotetext{
${ }^{1}$ See Gruen et al. (2002) for a detailed study on the incidence and consequences of out-of-stocks across different product categories and geographies.
} 
data on product availability. One would expect the extensive adoption of perpetual inventory management systems in retailing, which monitor inventory in real time, to alleviate this data limitation problem. However, there are two reasons why these inventory systems have not entirely solved this problem. First, in many cases these systems do not distinguish between inventory on the shelf or in the backroom, and as a consequence, the system might report availability for the store, although the shelf may be empty. Second, recent work by DeHoratius and Raman (2008) showed large discrepancies between the actual inventory and system-recorded inventory. Consequently, audits need to be conducted periodically to reconcile actual inventory with what is kept in the inventory system. Therefore, in practice, many retailers operate with a periodic inventory review system, where inventory is observed precisely only at specific time epochs and cannot be perfectly inferred at other points in time. For these reasons, we design our methods to work with sparse (partial) information of product availability, as provided by periodic inventory review systems.

Our estimation approach is based on an extension of the methodology in Musalem et al. $(2008,2009)$ for demand estimation from aggregate data. Accordingly, we treat the sequence of individual purchases in a given time period (i.e., the order in which individual purchases were made) as missing data and simulate these sequences from their posterior distribution. Combining these simulated sequences of purchases with periodic inventory information, we estimate the evolution of product inventory on the shelf and make inferences about the demand model, taking into account the occurrence of out-of-stocks. Consequently, we explicitly consider the endogenous variation in product availability, modeling the set of products available to a customer in a given time period as a function of the initial inventory and the sequence of choices made by customers. Moreover, when used in categories where out-of-stocks occur frequently, our data augmentation simplifies substantially the estimation of the model parameters relative to the expectation-maximization (EM) approach used in previous work (Anupindi et al. 1998, Conlon and Mortimer 2009). Furthermore, this structural demand approach to model product availability allows us to perform policy experiments that can be useful to estimate lost sales and evaluate the impact of policies designed to mitigate the consequences of out-ofstocks.

We illustrate how the model can be used to assist the decisions of a store manager in two ways. First, we estimate lost sales and substitution induced by out-of-stock products, which is used to assign a "financial" tag to out-of-stock events. Second, we use the model to evaluate the financial consequences of temporary price promotions that can help alleviate the costs of out-of-stocks by recapturing a fraction of the lost sales.

To summarize, our work makes four important contributions. First, we develop a methodology that can be applied in fairly general settings, including categories with a large number of products, some of which may be slow-moving products that exhibit zero sales in some periods. Second, our methodology explicitly considers the endogenous changes in product availability triggered by customer choices. Third, our structural demand approach allows us to perform policy experiments that can be useful to estimate lost sales and evaluate the impact of policies designed to reduce the consequences of out-of-stocks. Fourth, the use of data augmentation greatly simplifies the estimation of the model parameters, especially when compared with EM or maximum simulated likelihood approaches.

The rest of this paper is structured as follows. Section 2 relates our work to the existing literature. In $\S 3$, we describe the demand model, estimation methodology, and how the methodology can be used to estimate lost sales and stockout-based substitution. Section 4 presents an empirical application of the methodology using a data set on shampoo purchases. Section 5 estimates the costs of out-of-stocks and illustrates how the methodology can be used to assess the impact of different policies aimed at mitigating their financial consequences. Finally, $\S 6$ concludes this paper with a discussion of interesting avenues for future research.

\section{Literature Review}

Our work is related to research streams in the operations management, marketing, and economics literatures. For instance, our work is related to analytical models of inventory management and assortment planning developed in the operations management literature (e.g., Zipkin 2000, Smith and Agrawal 2000). These models typically assume a specific demand formulation that is incorporated into an optimization problem; but in general they provide little guidance on how to determine the input parameters of the demand model. This limits the applicability of this work in two ways. First, the demand model specification needs to be validated through empirical data for the prescribed decisions to be relevant in practice. Second, a naïve estimation of a correctly specified demand model that neglects the effect of out-of-stocks on sales can lead to biased estimates and incorrect model inputs and managerial recommendations. A notable exception in this literature is Kök and Fisher (2007), who estimate a demand model that captures the effect of permanent changes in an assortment on sales, which is then 
used to choose the number of facings of products to be included in an optimal assortment. Our focus is different in that we measure the effect of temporary changes of product availability on sales. ${ }^{2}$ Recent work by Vulcano et al. (2008) estimated substitution effects induced by out-of-stocks, using exact information on product availability. In contrast, our methodology can be used with partial information on product availability (as in a periodic review inventory system).

In the context of the marketing literature, models that use individual data (e.g., Fader and Hardie 1996, Rossi et al. 1996) or aggregate data (e.g., Besanko et al. 2003, Jiang et al. 2009) to estimate consumer demand are ubiquitous. However, most of this work ignores the effect of product availability on demand, probably because of the lack of data. ${ }^{3}$ Some studies use simple methods to infer availability from sales data, for example, by assuming that a product is out of stock when it has no sales (e.g., Campo et al. 2003, Swait and Erdem 2002). This approach is imprecise for categories with slow-moving products. Instead, we incorporate direct measures of product availability to estimate customers' preferences and reactions to stockouts.

To our knowledge, Anupindi et al. (1998) were the first to estimate the effect of out-of-stocks on customer demand using actual measures of product availability. Their model accounts for lost sales and product substitution effects, but unless further restrictions are imposed to this model, it is necessary to estimate different arrival rates for every possible set of available alternatives faced by a customer. Therefore, the number of parameters rapidly grows with the number of alternatives, and to fully characterize customers' propensity to buy each alternative, it is necessary to have observations for every possible choice set. ${ }^{4}$ To address this estimation issue, Anupindi et al. (1998) impose ad hoc restrictions on the substitution patterns. ${ }^{5}$ Kalyanam et al. (2007) model cross-item substitution through a finite number of categorical variables. This approach does not capture substitution through continuous measures such as price, and therefore cannot be used to compute price elasticities. In our model, substitution patterns are also restricted but based

\footnotetext{
${ }^{2}$ Kök and Fisher (2007) also develop a model to estimate stockoutbased substitution (temporary changes in availability), which they do not estimate with their empirical data.

${ }^{3}$ An exception is Anderson et al. (2006), who use sales data from a catalog retailer.

${ }^{4}$ Denoting by $J$ the number of alternatives, the number of nonempty choice sets is equal to $2^{J}-1$.

${ }^{5}$ More specifically, they assume "one-stage substitution," where a fraction of the demand for an out-of-stock product is transferred to a second product, but if that product is also out of stock, the demand is lost.
}

on a structural model of utility maximization, which enables us to account for price-based substitution.

In addition, our work is also related to the literature on consideration sets (e.g., Hauser and Wernerfelt 1990, Roberts and Lattin 1991, Andrews and Srinivasan 1995). This body of research focuses on modeling the set of alternatives that are considered by a consumer when estimating consumer preferences. In this paper, we face a similar problem because we are interested in estimating the underlying set of alternatives that are available to each customer. We estimate this set of alternatives combining aggregate sales data with information on product inventory.

In the economics literature there has been an extensive development of methods to estimate demand based on random utility maximization (RUM) models using market-level sales data (e.g., Berry 1994, Berry et al. 1995). RUM models can be very effective at providing a parsimonious characterization of consumer preferences, reducing the number of parameters to be estimated. In the context of out-of-stocks, Bruno and Vilcassim (2008) extend the methodology in Berry et al. (1995) to incorporate external information about product availability, showing that neglecting the effects of out-of-stocks leads to substantial biases in the estimation. As pointed out by Chintagunta and Dubé (2005), a limitation of the methodology of Berry (1994) and its extensions is that it cannot be used when some of the products have zero sales, which is not uncommon for store-level data of slow-moving categories. In contrast, our method can be used with store-level data and slow-moving products. Store-level data have the advantage of providing more precise information on which products are out of stock (relative to proxies of product availability that could be obtained at the market level).

Closest to our work is Conlon and Mortimer (2009), who estimate the substitution effects induced by stockouts using a random utility model and partial data on product availability. They use an EM algorithm to account for the missing data on product availability faced by each customer. However, the expectation step becomes difficult to implement when multiple products are simultaneously out of stock (as in the case of our empirical application). As we will explain in the next section, our approach can be applied to cases where a large number of products become out of stock without increasing the complexity of the estimation method. This enables us to uncover some interesting patterns describing how the number of out-of-stock products affects lost sales (the details are discussed in \$5).

In terms of Bayesian methods, our estimation approach is similar to the methods used by Chen and Yang (2007) and Musalem et al. (2008, 2009). In contrast to prior work in this area (e.g., Musalem 
et al. 2008), we not only use aggregate sales data, but also use periodic information about product inventory. Conditioning on both types of information, we jointly estimate the distribution of consumer preferences and product availability, which enables us to estimate the impact of out-of-stocks on consumer choices.

Finally, we note that other authors have used controlled laboratory experiments (e.g., Fitzsimons 2000), field experiments (e.g., Anderson et al. 2006), and questionnaires (e.g., Campo et al. 2000) to estimate customer response to out-of-stocks. We focus instead on developing methods that use field data routinely collected by store managers.

\section{Model and Methodology}

This section describes the customer choice model and the estimation method. The demand model is based on utility maximization principles and can be estimated with sales data from multiple stores combined with (partial) information about product availability. We also discuss identification and endogeneity issues and show results from a simulation experiment to test and validate our methodology. Finally, we show how the model can be used to estimate lost sales.

\subsection{Customer Choice Model}

We start by specifying a random-coefficients multinomial logit (MNL) model for product choice within a category, a demand specification widely used in the economics and marketing literature (e.g., Chintagunta et al. 1991, Train 2003). Consider a customer $i$ that visits store $m$ during time period $t$ and chooses to buy a single unit from among the alternatives in the set $\mathscr{F}=\{1, \ldots, J\}$ or chooses not to purchase (no-purchase option). We specify the utility of purchasing product $j \in \mathscr{F}$ as follows:

$$
U_{i j t m}=\beta_{i t m}^{\prime} x_{j t m}+\xi_{j t m}+\varepsilon_{i j t m},
$$

where $x_{j t m}$ is a vector of covariates that may include product characteristics, price, and other marketing variables. The vector of random coefficients $\beta_{i t m}$, which varies across customers, describes individual preferences and is assumed to be distributed according to a multivariate normal with mean $\bar{\theta}_{m}$ and covariance matrix $\Sigma$. Note that the vector of preference coefficients $\beta_{i t m}$ has $i, t$, and $m$ subscripts, which allows different customers visiting a store in each period to have different preference coefficients, albeit drawn from the same distribution. ${ }^{6}$ The mean of individual preferences for a given store $m$ is specified as

$$
\bar{\theta}_{m}=\theta \cdot z_{m}
$$

${ }^{6}$ This corresponds to the independent samples assumption described in Musalem et al. (2009), where a different random sample of consumers make purchase decisions in each period. Musalem et al. (2009) also show that this assumption is asymptotically equivalent to the case where the same consumers make purchase decisions in every time period, i.e., $\beta_{i t m}=\beta_{i m}$. where $z_{m}$ is a vector of time-varying characteristics for store $m$, which enables a researcher to capture observed heterogeneity across customers in different markets and $\theta$ is a matrix of coefficients. The variancecovariance matrix $\Sigma$ captures unobserved customer heterogeneity, which is assumed for simplicity and parsimony to be constant across stores. The term $\xi_{j t m}$ is a demand shock for each product $j$, common to all customers, that represents other factors that enter customer's utility that are not captured by $x_{j t m}$. We also write $X_{t m}=\left(x_{1 t m}, \ldots, x_{J t m}\right)$ and $\xi_{t m}=\left(\xi_{1 t m}, \ldots, \xi_{J t m}\right)$ to describe the product characteristics and demand shocks of all products in store $m$ during period $t$. The random vectors $\xi_{t m}$ are independent and identically distributed (i.i.d.) samples of a multivariate normal distribution with zero mean and covariance matrix $\Sigma_{\xi}$. For simplicity, we assume that $\Sigma_{\xi}=\sigma_{\xi}^{2}$. $I_{J}$ (where $I$ is the identity matrix with $J$ rows and columns). The inclusion of these demand shocks helps to prevent overfitting problems when aggregate data are used to estimate the model (Berry 1994). Finally, $\varepsilon_{i j t m}$ is an individual-specific demand shock, modeled as an i.i.d. random variable from an extreme value distribution.

The probability that a customer chooses a particular brand during a given time period depends on the set of alternatives available to a customer. In particular, because of the occurrence of out-of-stocks, the choice set of a customer may not include all the products in the set $\mathscr{F}$. Product availability is characterized in our model by the vector $a_{i t m}=\left(a_{i t m}^{1}, \ldots, a_{i t m}^{J}\right)$, where $a_{i t m}^{j}=1$ if customer $i$ visiting store $m$ in period $t$ finds product $j$ available, and $a_{i t m}^{j}=0$ otherwise. Without any loss of generality, we index customers in each period by their order of arrival to the store. The matrix $A_{t m}=\left(a_{1 t m}, \ldots, a_{N_{m} t m}\right)$ contains the unobserved product availability information for all customers visiting that store in period $t$, which will be structurally inferred.

Let $y_{\text {itm }}$ denote the product chosen by customer $i$ in period $t$ and market $m$, and let $U_{i 0 t m}=\varepsilon_{i 0 t m}$ be the utility of the no-purchase option (the subscript 0 denotes the no-purchase option). The probability that a customer $i$ facing availability $a_{i t m}$ purchases product $j$ is given by

$$
\begin{aligned}
& p_{j}\left(\beta_{i t m}, \xi_{t m} \mid a_{i t m}, X_{t m}\right) \\
& \quad \equiv \operatorname{Pr}\left(y_{i t m}=j \mid \beta_{i t m}, a_{i t m}, \xi_{t m}, X_{t m}\right) \\
& =\frac{a_{i t m}^{j} \cdot \exp \left(\beta_{i t m} X_{j t m}+\xi_{j t m}\right)}{1+\sum_{k \in \mathcal{F}} a_{i t m}^{k} \cdot \exp \left(\beta_{i t m} X_{k t m}+\xi_{k t m}\right)},
\end{aligned}
$$

whereas the probability of choosing the no purchase option is given by $p_{0}\left(\beta_{i t m}, \xi_{t m} \mid a_{i t m}, X_{t m}\right)=$ $1-\sum_{j \in \mathscr{F}} p_{j}\left(\beta_{i t m}, \xi_{t m} \mid a_{i t m}, X_{t m}\right)$. Note that this customer model is similar to the one used by Bruno and 
Vilcassim (2008) and Conlon and Mortimer (2009) and represents an extension to random coefficients to handle (observed or unobserved) product availability. ${ }^{7}$

\subsection{Derivation of the Likelihood Function of the Aggregate Data}

As is evident from Equation (3), computing the probability of purchase requires information about the choices of each customer visiting the store $\left(y_{i t m}\right)$ and the set of products available to each of them $\left(a_{i t m}\right)$. As we mentioned in the introduction, this information is not always available in practice. Therefore, we seek to estimate the parameters of the customer choice model, $\left(\beta, \Sigma, \sigma_{\xi}\right)$, using the following aggregate data: (1) sales of each product for each store period, $S_{j t m}$; (2) inventory at the store at the beginning and end of each period, $I_{j t m}$ and $\hat{I}_{i t m}$, respectively; and (3) the number of customers making purchase decisions in each period, $N_{t m} .{ }^{8}$ Information about $N_{t m}$ can be obtained, for example, from the total number of transactions recorded for each store in each period. Alternatively, it is also possible to use demographic information (e.g., population data) to estimate the size of the market (i.e., the maximum number of consumers that would purchase any of the alternatives in the product category in a given time period; see Berry et al. 1995). The number of customers choosing the no-purchase option is then given by $S_{0 t m} \equiv$ $N_{t m}-\sum_{j=1}^{J} S_{j t m}$. If there are replenishments, the definition of the time periods is such that they occur just before the beginning of the period and are therefore accounted in $I_{t m}$.

Note that when a store $m$ runs out of stock for some product $j$ during period $t$, we observe that $I_{t m}^{j}>0$ and $\hat{I}_{t m}^{j}=0$ but we do not know the exact time the product went out of stock. This missing piece of information is important because it determines how many customers were exposed to the out-of-stock. For example, if the first customer visiting the store purchased the last unit of product $j$, then all other customers were exposed to this out-of-stock. In contrast, if the last customer visiting the store purchased the last unit of product $j$, then no customers were affected by this out-of-stock. Because this information is not directly observable, we do not know a priori whether customers buying product $k \neq j$ during that period chose this product because it was their most preferred item or because it was their second preferred option when product $j$, the preferred product, was not available.

\footnotetext{
${ }^{7}$ Campo et al. (2003) make use of a similar extension to the MNL model, but do not incorporate random coefficients.

${ }^{8}$ Note that store sales are usually monitored more frequently than product availability. In those cases, our method can still be applied after aggregating sales to form a sales series with the same frequency as the availability data.
}

Consequently, given that the purchasing probability depends on the availability vector $a$, the likelihood function for this problem cannot be expressed only in terms of sales data and the coefficients of the utility function of each customer, as in standard applications of choice models to purchase data.

These difficulties caused by sparse product availability data were recognized by Anupindi et al. (1998) and Conlon and Mortimer (2009). They develop a model where the time epoch of each out-of-stock is treated as missing data, and use an EM algorithm for estimation. The EM algorithm facilitates the search for the maximum likelihood (ML) estimators. However, the closed-form expressions used in their expectation step become complicated as the number of out-ofstocks in a single period increases. ${ }^{9}$

Our methodology instead uses a data augmentation approach to incorporate incomplete data on product availability into the estimation. This approach circumvents some of the limitations of other methods, in particular, it can be easily implemented when some periods have multiple stockouts. These augmented data are described as follows. Let $w_{i t m}^{j}$ be a choice indicator equal to 1 if $y_{i t m}=j$, and let $w_{i t m}$ be a row vector containing these indicators (defined similarly as $\left.a_{i t m}\right)$. The matrix $W_{t m}=\left(w_{1 t m}, \ldots, w_{N_{m} t m}\right)$ contains all of the choice indicators corresponding to period $t$ and market $m$. A key element of our approach is that $W_{t m}$, sales $S_{t m}$, and the initial and ending inventories $\left(I_{t m}, \hat{I}_{t m}\right)$ uniquely determine the set of products available to each customer, $a_{i t m}$, through the following structural relationships:

$$
a_{i t m}^{j}= \begin{cases}1 & \text { if } I_{t m}^{j}>0, \hat{I}_{t m}^{j}>0, \\ 0 & \text { if } I_{t m}^{j}=\hat{I}_{t m}^{j}=0, \\ 1\left\{\sum_{k=1}^{i-1} w_{k t m}^{j}<S_{j t m}\right\} & \text { if } I_{t m}^{j}>0, \hat{I}_{t m}^{j}=0 .\end{cases}
$$

The augmented data are defined by $A_{t m}$ and $W_{t m}$ for all stores and periods, whereas the observed data are given by $\left(S_{t m}, I_{t m}\right)$ (the ending inventory vector $\hat{I}_{t m}$ can be obtained as $I_{t m}-S_{t m}$ and is therefore redundant). The augmented data $\left(A_{t m}, W_{t m}\right)$ are exactly consistent with the observed data if they satisfy Equation (4) and if

$$
\begin{gathered}
\sum_{i=1}^{N_{t m}} w_{i j t m}=S_{j t m} \quad \text { for all } j \in \mathscr{F}, \\
w_{i t m}^{j} \leq a_{i t m}^{j} .
\end{gathered}
$$

${ }^{9}$ Anupindi et al. (1998) derive expressions for no more than two out-of-stocks taking place in the same period. Conlon and Mortimer (2009) provide simulation techniques to approximate the expectation step when multiple stockouts occur in the same period. 
Accordingly, let $\Omega_{t m}\left(S_{t m}, \hat{I}_{t m}\right)$ be the set containing all values of $A_{t m}, W_{t m}$ that are consistent with the observed data (defined by (4), (5), and (6)).

Our data augmentation approach can be illustrated with the following example. Suppose that two products, $\mathrm{B}$ and $\mathrm{C}$, are available at the beginning of a given time period, but only product $C$ is available at the end of the period. We observe total period sales for each product: $S_{\mathrm{B}}=1$ and $S_{\mathrm{C}}=2$ (we suppress indexes $t$ and $m$ for convenience). Therefore, we know that initially there was exactly $I_{\mathrm{B}}=1$ unit of $\mathrm{B}$ and at least $I_{C}=3$ units of $C$ in inventory. Furthermore, suppose we also observe that $N=6$ customers visited the store during this period; therefore, $N-S_{B}-S_{C}=3$ customers chose the no-purchase option, denoted by 0 . We index customers by their order of arrival and the choice of each customer by $y_{i} \in\{B, C, 0\}$. The vector $y=\left(y_{1}, \ldots, y_{6}\right)$ characterizes the order of purchases and determines the choice indicators $w_{i j}$. If $y=(C, 0, C, 0,0, B)$, then all six customers found both products available. If we consider instead a different value of the vector of individual choices by swapping the choices of customers 1 and 6 , we obtain $y=(B, 0, C, 0,0, C)$. In this case, customers 2-6 found product $B$ out of stock. Also note that had we swapped instead the choices of customers 1 and 2, $y=(0, C, C, 0,0, B)$, then the set of products available to each customer would be unchanged.

We now derive the likelihood function in several steps. All the expressions below are conditional on $X$ and $Z$, which we omit to simplify the notation. First, note that the likelihood of the observed data can be expressed in terms of the likelihood of the augmented data using the law of total probability:

$$
\begin{aligned}
& L_{t m}\left(\theta, \Sigma, \Sigma_{\xi} \mid S_{t m}, I_{t m}\right) \\
& \quad=\sum_{\left(W_{t m}, A_{t m}\right) \in \Omega_{t m}\left(S_{t m}, I_{t m}\right)} L_{t m}\left(\theta, \Sigma, \Sigma_{\xi} \mid W_{t m}, A_{t m}\right) .
\end{aligned}
$$

Second, the likelihood conditional on the augmented data $W_{t m}, A_{t m}$ can be computed conditioning on $\beta_{i t m}$ and $\xi_{t m}$ and integrating over their distribution:

$$
\begin{aligned}
& L_{t m}\left(\theta, \Sigma, \Sigma_{\xi} \mid A_{t m}, W_{t m}\right) \\
& =\int_{\xi_{t m}} \prod_{i=1}^{N_{m}} \int_{\beta_{i t m}} L_{i t m}\left(\beta_{i t m}, \xi_{t m} \mid w_{i t m}, a_{i t m}\right) \\
& \quad \cdot \phi\left(\beta_{i t m} ; \theta^{\prime} Z_{m}, \Sigma\right) \phi\left(\xi_{t m} ; 0, \Sigma_{\xi}\right) d \beta_{i t m} d \xi_{t m},
\end{aligned}
$$

where $L_{i t m}\left(\beta_{i t m}, \xi_{t m} \mid w_{i t m}, a_{i t m}\right)$ is the likelihood for an individual customer as a function of her preference coefficients $\beta_{i t m}$ and the demand shock $\xi_{t m}$. Using the individual choice probability defined in (3), this likelihood can be calculated as

$$
L_{i t m}\left(\beta_{i t m}, \xi_{t m} \mid w_{i t m}, a_{i t m}\right)=\prod_{j=0}^{J} p_{j}\left(\beta_{i t m}, \xi_{t m} \mid a_{i t m}\right)^{w_{i t m}^{j}}
$$

Equations (7), (8), and (9) completely characterize the likelihood for store $m$ and period $t$ in terms of the observed data. Consequently, the total likelihood (including all stores and periods) is given by

$$
L\left(\theta, \Sigma, \Sigma_{\xi} \mid S, I\right)=\prod_{m=1}^{M} \prod_{t=1}^{T} L_{t m}\left(\theta, \Sigma, \Sigma_{\xi} \mid S_{t m}, I_{t m}, X_{t m}\right)
$$

This likelihood function is difficult to compute because of the summation over all possible configurations of choice indicators $\left(W_{t m}\right)$ exactly consistent with the aggregate data (see Equation (7)). ${ }^{10}$ Instead of using an ML approach, our strategy is to apply data augmentation together with Markov chain Monte Carlo (MCMC) methods to facilitate the estimation of the parameters of the model. Accordingly, we will treat the augmented data $\left(W_{t m}, A_{t m}\right)$ as model parameters that are estimated using MCMC simulation. This approach is described in the next subsection.

\subsection{Bayesian Estimation}

In contrast to an ML approach, which seeks to find a point estimate for the parameters of interest $\left(\theta, \Sigma, \Sigma_{\xi}\right)$, a Bayesian approach seeks to estimate the posterior distribution for the parameters, given the observed data. Toward this objective, we define the hyperprior distribution $\pi\left(\theta, \Sigma, \Sigma_{\xi}\right)$, which formalizes the researcher's prior beliefs about the demand parameters. The posterior density of $\left(\theta, \Sigma, \Sigma_{\xi}\right),\left\{\beta_{i t m}\right\}$, and the augmented data $\left(A_{t m}, W_{t m}\right)$ given the observed data is proportional to

$$
\begin{aligned}
p\left(\theta, \Sigma, \Sigma_{\xi},\left\{A_{t m}\right\},\left\{W_{t m}\right\},\left\{\beta_{i t m}\right\} \mid S, I\right) \\
\propto \prod_{t} \prod_{m} 1\left\{\left(A_{t m}, W_{t m}\right) \in \Omega\left(S_{t m}, I_{t m}\right)\right\} \phi\left(\xi_{t m} ; 0, \Sigma_{\xi}\right) \\
\quad \times \prod_{i=1}^{N_{t m}} \phi\left(\beta_{i t m} ; \theta^{\prime} Z_{m}, \Sigma\right) \\
\quad \cdot L_{i t m}\left(\beta_{i t m}, \xi_{t m} \mid a_{i t m}, w_{i t m}\right) \pi\left(\theta, \Sigma, \Sigma_{\xi}\right),
\end{aligned}
$$

where the indicator function ensures that the augmented data are exactly consistent with the observed data.

We seek to estimate the posterior (10) using MCMC methods. Conditional on the augmented variables $(a, w)$, the parameters $\left\{\beta_{i t m}\right\}, \theta, \Sigma$, and $\Sigma_{\xi}$ can be sampled using existing Bayesian methods for individual level data (e.g., Allenby and Rossi 2003). In particular, we combine these existing methods with a sampling scheme for the missing data $A$ and $W$. Following Musalem et al. (2009), posterior samples

\footnotetext{
${ }^{10}$ Another complication of implementing a maximum likelihood approach is that the integrals in Equation (8) cannot be expressed in closed analytical form and have to be approximated through simulation.
} 
Table 1 Gibbs Sampling Example

\begin{tabular}{lcccccc}
\hline Customer $i$ & $y_{i}$ & $a_{i}^{\mathrm{B}}$ & $a_{i}^{\mathrm{C}}$ & $y_{i}^{*}$ & $a_{i}^{\mathrm{B} *}$ & $a_{i}^{\mathrm{C} *}$ \\
\hline 1 & $\mathrm{C}$ & 1 & 1 & $\mathrm{C}$ & 1 & 1 \\
2 & 0 & 1 & 1 & $\mathrm{~B}$ & 1 & 1 \\
3 & $\mathrm{C}$ & 1 & 1 & $\mathrm{C}$ & 0 & 1 \\
4 & $\mathrm{~B}$ & 1 & 1 & 0 & 0 & 1 \\
5 & 0 & 0 & 1 & 0 & 0 & 1 \\
6 & 0 & 0 & 1 & 0 & 0 & 1 \\
\hline
\end{tabular}

for these variables can be obtained by deriving the full-conditional distribution of the augmented choices and availability data. Note that the full-conditional distributions have a simple structure if we partition the set of customers into pairs and consider the distribution of the choices of customers in a given pair, holding constant all other choices. ${ }^{11}$ This enables us to define a direct Gibbs mechanism to sequentially sample $a$ and $w$ from their posterior distribution for each pair of customers in contrast with other methods that utilize Metropolis-Hastings approaches (e.g., Chen and Yang 2007). We illustrate this sampling scheme with an example (see Table 1).

To simplify notation, we suppress time $(t)$ and store $(m)$ indexes for this example. Consider the set of products formed by $\{\mathrm{B}, \mathrm{C}\}$ plus the no-purchase option 0 . The observed data are $S_{\mathrm{B}}=1, S_{\mathrm{C}}=2, N=6$, $I^{\mathrm{B}}=1$, and $I^{\mathrm{C}}=3$ (which implicitly define the end-ofperiod inventories $\hat{I}^{\mathrm{B}}=0$ and $\left.\hat{I}^{\mathrm{C}}=1\right)$. We start with an initial assignment of choices given by $y$ (see the second column in Table 1), which is consistent with the observed sales data. We now show how to sample a new vector $y^{\prime}$. We randomly generate a partition of the set of customers into three pairs: for example, $\{1,3\},\{2,4\},\{5,6\}$. We are interested in calculating the full-conditional distribution of the choices of customers in one of these pairs, say $\{2,4\}$. Conditioning on the choices of all other customers $\{1,3,5,6\}$, denoted by $y_{-24}$, and the observed data, there are only two vectors of choices that are consistent with the sales data: the current choices $(y)$ and the choice vector $y^{*}$ (see the fifth column in Table 1) where the choices of customers 2 and 4 are swapped.

Note that when exchanging the choices of customers 2 and 4 , the sets of available products to customers arriving between customers 2 and 4 may endogenously change (hence the structural estimation of out-of-stocks), but the choice set of all other customers remains unchanged. In the example, customers 2, 3, and 4 find alternative B available under the current configuration of choices $(y$, see the third

\footnotetext{
${ }^{11}$ As mentioned in Musalem et al. (2009), one could also consider partitioning the set of customers into larger groups (e.g., triplets or quadruplets). This may provide a more efficient approach to estimate the posterior distribution of the model parameters, but it increases the computational cost of each simulation.
}

column in Table 1), whereas only customer 2 finds this alternative available under the new configuration ( $y^{*}$, see column 6 in Table 1). In addition, product availability for all other customers (1, 5, and 6) remains constant. Therefore, swapping the choices of customers 2 and 4 can only affect the likelihood of customers 2,3 , and $4 .{ }^{12}$ When sampling from this fullconditional distribution, the probability of generating a draw where the choices of 2 and 4 are exchanged is

$$
\begin{aligned}
& \operatorname{Pr}\left(y^{*} \mid y_{-\{2,4\}}\right) \\
& \quad=\frac{\prod_{i=2}^{4} L_{i}\left(\beta_{i}, \xi \mid a_{i}^{*}, w_{i}^{*}\right)}{\prod_{i=2}^{4} L_{i}\left(\beta_{i}, \xi \mid a_{i}^{*}, w_{i}^{*}\right)+\prod_{i=2}^{4} L_{i}\left(\beta_{i}, \xi \mid a_{i}, w_{i}\right)}
\end{aligned}
$$

where $a^{*}, w^{*}$ is the augmented data implied by the vector of choices $y^{*}$, and $L_{i}\left(\beta_{i}, \xi \mid a_{i}^{*}, w_{i}^{*}\right)$ is the individual likelihood defined in (9). The new draw is equal to the previous draw $y$ with probability $1-\operatorname{Pr}\left(y^{*} \mid y_{-\{2,4\}}\right)$.

Based on this intuition, the Gibbs sampling scheme for the missing data $\left(A_{t m}, W_{t m}\right)$ of each store period can be formalized as follows.

Step 1 . In a given iteration $r$, randomly generate a partition of customer pairs, denoted $P_{t m}$, of the $N_{t m}$ customers.

Step 2. For a given pair $(l, k) \in P_{t m}$, exchange the choices of customers $l$ and $k$. Generate the new choice vector $y_{t m}^{*}$ and the corresponding choice indicators $W_{t m}^{*}$ and availability matrix $A_{t m}^{*}$. The new variables are accepted with probability

$\operatorname{Pr}($ accept $)$

$=\frac{\prod_{i=l}^{k} L_{i t m}\left(\beta_{i t m}, \xi_{t m} \mid a_{i t m}^{*}, w_{i t m}^{*}\right)}{\prod_{i=l}^{k} L_{i}\left(\beta_{i t m}, \xi_{t m} \mid a_{i t m}^{*}, w_{i t m}^{*}\right)+\prod_{i=l}^{k} L_{i}\left(\beta_{i t m}, \xi_{t m} \mid a_{i t m}, w_{i t m}\right)}$.

If accepted, update $y_{t m}^{(r+1)} \leftarrow y_{t m}^{*}, a_{t m}^{(r+1)} \leftarrow a_{t m}^{*}$, and $w_{t m}^{(r+1)} \leftarrow w_{t m}^{*}$, otherwise set $y_{t m}^{(r+1)} \leftarrow y_{t m}^{(r)}, a_{t m}^{(r+1)} \leftarrow a_{t m}^{(r)}$, and $w_{t m}^{(r+1)} \leftarrow w_{t m}^{(r)}$. Repeat Step 2 for a new pair in $P_{t m}$ until no pairs are left.

The Gibbs sampling scheme ensures that every feasible choice vector $y$ can be sampled with positive probability. Therefore, when combined with a proper sampling scheme for $\left\{\beta_{i t m}\right\},\left\{\xi_{t m}\right\}, \theta$, and $\Sigma$, it generates an irreducible Markov chain with stationary probability distribution equal to the posterior distribution of the parameters of the model.

\subsection{Identification and Assumptions}

In this subsection, we discuss issues related to identification of our model. In the economics and marketing literature, variations in prices, promotions, and other

\footnotetext{
${ }^{12}$ In general, only customers within the range of the swapped choices can be affected, which provides significant computational savings.
} 
marketing variables are usually exploited to identify and estimate demand models (e.g., Villas-Boas and Winer 1999). In this paper, another important source of identification arises from changes in product availability. In particular, observing customer behavior under different choice sets provides useful information to uncover the patterns of substitution among different alternatives. One difficulty is that the variation in product availability is not perfectly observed and this may lead to some challenges in the identification of the model parameters.

Consider the following example. A few units of product $\mathrm{A}$ are available at the beginning of a period, and at the end of period the product is out of stock. There are two possible explanations that fit these data. In the first one, customers have a low utility for product A (relative to other products), which generates just enough demand for all the units initially available. In this case, the out-of-stock of product A occurs toward the end of the period. A second explanation is that customers place a high utility on product $\mathrm{A}$, and the first customers visiting the store purchase product $\mathrm{A}$, generating an out-of-stock early in the period. Without additional information, we cannot distinguish between these two alternative explanations. However, we may also observe data for (1) the sales for other products with similar characteristics to product A, which were available throughout the period; and (2) sales during other periods where product A had full availability. This additional information is useful to separately identify the utility of product $\mathrm{A}$ and the latent distribution of product availability. ${ }^{13}$

Although periodic inventory data provides sufficient information on product availability to estimate the model, the estimation could still be carried out without the exact inventory counts. More specifically, Equation (4) requires information on product availability-whether a product is available or not-at the beginning and end of each period, but the actual inventory counts are not required for the estimation. This is important, because it has been documented that inventory count data are not always reliable in practice (e.g., DeHoratius and Raman 2008). Tracking product availability is less costly than

\footnotetext{
${ }^{13}$ The demand model could also be estimated by considering only the time periods for which the set of available alternatives does not change, i.e., periods in which the set of products available at the beginning and end of the period is the same. Although this would certainly reduce the complexity of the estimation method, this approach exhibits several disadvantages. First, there is a sample selection problem, because periods where a product becomes unavailable are likely to have higher than average demand. Second, one would be forced to discard valuable information about the customer demand, and this approach would only work if there are enough time periods that meet this requirement. In particular, for longer time periods, it is more likely to have some products that stock out during the period.
}

tracking inventory counts, and retailers often run periodic inspections of shelves to check which products are out of stock.

An additional challenge that we face in the estimation of our model is related to the potential endogeneity of inventory. Inventory is a supply decision made by managers that usually requires forecasting of future demand (e.g., see Cachon and Terwiesch 2005). Mismatches between supply and demand, which lead to stockouts or excess inventory, suggest that managers cannot predict demand perfectly, i.e., there is demand uncertainty from the managers' point of view. Our model captures this demand uncertainty through the demand shock $\xi_{j t m}$, which accounts for unpredictable factors (from the managers' perspective) that determine actual demand. However, in some cases it may be difficult to obtain all the relevant demand information that was accounted for in the inventory decision. For example, some retail chains decentralize inventory decisions to store managers to take advantage of local demand information (see O'Connell 2008). However, this local information may not be easy to transfer to a central analyst of the chain who is using our model to conduct demand estimation. In this case, the demand shock $\xi_{j t m}$ would also include factors that are observed by the store manager (e.g., information about local demand patterns) but are not available to the central demand analyst. Hence, $I_{j t m}$ and $\xi_{j t m}$ may not be independent, which leads to an endogeneity problem. As pointed out by Manchanda et al. (2004), conducting the estimation using the conditional likelihood (9), which ignores the dependency of inventories on the unobservable components of the demand, could lead to biased estimates.

To mitigate the potential endogeneity bias, our empirical application includes covariates measuring seasonal demand variation and promotional activity, which capture most of the demand shifts that could be anticipated by the store manager (the data set is described in more detail in \$4). In addition, it is possible to use a Bayesian instrumental variable (IV) approach similar to the one developed by Yang et al. (2003) to capture the dependency of the inventory levels on demand shocks mitigating a potential endogeneity bias. Specifically, the demand system could be estimated simultaneously with an additional regression equation specified as

$$
\ln \left(1+I_{j t m}\right)=\gamma_{x} X_{j t m}+\gamma_{z} Z_{j t m}+\nu_{j t m}
$$

where $Z_{j t m}$ is a vector of instrumental variables that are excluded from the demand model and orthogonal to the demand shock $\xi_{i t m}$. Examples of valid instruments include factors that affect inventory holding costs (such as capital costs or store rent), frequency and lead times of replenishments (e.g., distance to 
warehouse), and factors that affect target service levels (e.g., cost markups; see Cachon and Olivares 2010). To account for inventory endogeneity, $\xi_{j t m}$ and $v_{j t m}$ should be allowed to be correlated, for example, by assuming a bivariate normal distribution with zero mean and covariance matrix $\Omega_{\xi, \nu}$. Equation (12) could then be incorporated into the likelihood function (9), estimating the parameters $\gamma_{x}, \gamma_{z}$, and $\Omega_{\xi, \nu}$ jointly with the parameters characterizing the demand system. When the off-diagonal terms of this covariance matrix are nonzero, the distribution of $I_{j t m}$ conditional on $X, Z$ depends on $\xi_{j t m}$, thereby capturing the dependency of inventory levels on potential unobservable factors entering the store manager's inventory decision. Further details on this instrumental variable approach using hierarchical Bayes methods can be found in Yang et al. (2003).

As we discuss in $\$ 4$, our empirical results show that the demand parameters obtained from this joint estimation are almost identical to those obtained with the demand system alone. Nevertheless, extending the model to account for a potential dependency between inventories and demand shocks (through Equation (12)) could be relevant in other applications where the information used by the store manager to predict demand is not available to the researcher.

In the abscence of instruments, it is possible to follow an approach similar to that of Manchanda et al. (2004) to account for inventory endogeneity. For example, we can assume that each demand shock $\xi_{i t m}$ is distributed according to a Normal distribution with mean $\bar{\xi}_{j m}$ and variance $\sigma_{\xi}^{2}$, where the means are treated as random coefficients. If we replace $Z_{j t m}$ by $\bar{\xi}_{j m}$ in Equation (12) and assume that $\operatorname{Cov}\left(v_{j t m}, \xi_{j t m}-\bar{\xi}_{j m}\right)=0$, our model becomes a special case of the model developed by Manchanda et al. (2004), which can be estimated with the Bayesian techniques described therein.

Finally, another potential endogeneity problem may arise from serially correlated demand shocks $\left(\xi_{i t m}\right)$. Today's inventory depends on yesterday's demand, which induces a correlation between current inventory and previous demand shocks. In addition, if demand shocks are autocorrelated, current demand shocks will be correlated with previous shocks that are in turn correlated with today's initial inventory, leading to an endogeneity problem. As we previously mentioned, we do not expect to observe autocorrelation in demand shocks in our application once we control for seasonality. Nevertheless, the methodology presented here could be extended to allow for demand shock persistence, which would eliminate this form of endogeneity.

\subsection{Numerical Experiment}

In this subsection, we test the proposed methodology using simulated data. We generated sales and inventory data for $J=10$ purchase alternatives and a no-purchase option available in $M=12$ markets and $T=15$ periods. We included four variables $\left(x_{1}, \ldots, x_{4}\right)$ in the utility function. We use random coefficients for $x_{3}$ and $x_{4}$ and assume fixed (i.e., constant across customers in a given market) coefficients for $x_{1}$ and $x_{2}$. The first two variables correspond to two brands dummies, one for alternatives 1,2 , and $3\left(x_{1}\right)$, and another for alternatives 4,5 , and $6\left(x_{2}\right)$. The third variable is a dummy variable equal to 1 for all purchase alternatives $\left(x_{3}\right)$, whereas the fourth variable is generated from a normal distribution $\left(x_{4}\right)$. To replicate some of the features of the data set used in our empirical application, the continuous variable is generated so that its values for a given brand and market are the same across all time periods. Accordingly, the values of $x_{4}$ for each brand and market are generated from a normal distribution with mean equal to 2 and variance equal to 1 .

Based on these four explanatory variables, customer coefficients for a given market $m$ are generated from a four-dimensional multivariate normal distribution with mean $\bar{\theta}_{m}=\theta^{\prime} Z_{m}$ as in Equation (2) and variance $\Sigma$, where $Z_{m}$ represents a two-dimensional vector of demographic variables. In terms of the demographics, the first variable $z_{1 m}$ is equal to 1 for all markets (intercept), whereas the second variable $z_{2 m}$ is generated from a uniform distribution in the interval $[-1.5,1.5]$. The true value of $\theta$ corresponds to

$$
\theta=\left[\begin{array}{rrrr}
2.0 & 1.5 & -3.0 & -2.5 \\
0.5 & -0.5 & 0.0 & 0.7
\end{array}\right]
$$

The variance of the random coefficients $(\Sigma)$ is equal to a diagonal matrix with elements equal to 0.8 and 2.0 for $x_{3}$ and $x_{4}$, respectively, and elements equal to zero for the two variables with fixed coefficients $\left(x_{1}\right.$ and $\left.x_{2}\right)$. The size of each market $\left(N_{m}\right)$ is generated by taking the integer part of a uniform random variable defined on the interval $[0,300]$. In addition, common demand shocks for each alternative in each period and market $\left(\xi_{j t m}\right)$ are generated from a normal random variable with zero mean and variance $\sigma_{\xi}^{2}$ equal to 0.5. Finally, initial inventory levels for each alternative are generated by taking the integer part of a uniform random variable in the interval $[0, I]$. We use three different values of $I(10,60$, and 400), which lead to different inventory service levels. In the first case $(I=10)$, each alternative stocks out, on average, in $28.9 \%$ of the time periods across all markets, whereas this fraction corresponds to $8.7 \%$ and $1.3 \%$ for the second and third cases, respectively.

For each of these three data sets $(I=10, I=60$, and $I=400$ ) we estimated $\theta, \Sigma$, and $\sigma_{\xi}^{2}$ using the method described in $\$ 3.3$ and based on the aggregate data available for each period (sales and initial inventory). We also estimated these parameters ignoring 
Musalem et al.: Structural Estimation of the Effect of Out-of-Stocks

Table 2 Results: Estimated Posterior Means, Standard Deviations, and Quantiles for $\theta, \Sigma$, and $\sigma_{\xi}^{2}$, Ignoring (Benchmark Model) and Accounting for (Full Model) the Occurrence of Out-of-Stocks

\begin{tabular}{|c|c|c|c|c|c|c|c|c|c|c|c|c|}
\hline \multirow[b]{2}{*}{ Model } & & \multicolumn{4}{|c|}{ Main effects $\left(\theta_{z_{1}},\right)$} & \multicolumn{4}{|c|}{ Demographic interaction $\left(\theta_{z_{2}},\right)$} & \multicolumn{2}{|c|}{ Heterogeneity $(\Sigma)$} & \multirow[b]{2}{*}{$\sigma_{\xi}^{2}$} \\
\hline & & Brand 1 & Brand 2 & Intercept & Covariate & Brand 1 & Brand 2 & Intercept & Covariate & Intercept & Covariate & \\
\hline \multirow{5}{*}{$\begin{array}{c}\text { Benchmark } \\
I=10\end{array}$} & Mean & 1.45 & 1.30 & -4.13 & -1.52 & 0.41 & -0.39 & 0.00 & 0.53 & 0.66 & 0.93 & 0.48 \\
\hline & SD & 0.09 & 0.09 & 0.22 & 0.22 & 0.09 & 0.10 & 0.10 & 0.06 & 0.39 & 0.24 & 0.06 \\
\hline & $2.5 \%$ & 1.28 & 1.14 & -4.60 & -1.96 & 0.23 & -0.59 & -0.19 & 0.43 & 0.24 & 0.54 & 0.36 \\
\hline & $50.0 \%$ & 1.45 & 1.30 & -4.11 & -1.49 & 0.41 & -0.39 & 0.01 & 0.53 & 0.49 & 0.90 & 0.48 \\
\hline & $97.5 \%$ & 1.62 & 1.48 & -3.78 & -1.13 & 0.60 & -0.18 & 0.19 & 0.65 & 1.58 & 1.46 & 0.60 \\
\hline \multirow{5}{*}{$\begin{array}{c}\text { Benchmark } \\
I=60\end{array}$} & Mean & 1.82 & 1.37 & -3.39 & -2.14 & 0.41 & -0.50 & -0.02 & 0.70 & 0.85 & 1.56 & 0.61 \\
\hline & SD & 0.07 & 0.08 & 0.09 & 0.12 & 0.10 & 0.10 & 0.09 & 0.06 & 0.24 & 0.19 & 0.05 \\
\hline & $2.5 \%$ & 1.68 & 1.22 & -3.58 & -2.38 & 0.22 & -0.71 & -0.18 & 0.59 & 0.47 & 1.23 & 0.52 \\
\hline & $50.0 \%$ & 1.82 & 1.37 & -3.40 & -2.13 & 0.41 & -0.50 & -0.02 & 0.70 & 0.82 & 1.54 & 0.61 \\
\hline & $97.5 \%$ & 1.96 & 1.54 & -3.21 & -1.94 & 0.59 & -0.30 & 0.14 & 0.81 & 1.41 & 1.95 & 0.72 \\
\hline \multirow{5}{*}{$\begin{array}{c}\text { Benchmark } \\
I=400\end{array}$} & Mean & 1.94 & 1.70 & -3.09 & -2.54 & 0.38 & -0.62 & 0.06 & 0.71 & 0.52 & 2.04 & 0.60 \\
\hline & SD & 0.09 & 0.09 & 0.11 & 0.11 & 0.09 & 0.09 & 0.08 & 0.04 & 0.22 & 0.17 & 0.05 \\
\hline & $2.5 \%$ & 1.77 & 1.52 & -3.32 & -2.76 & 0.21 & -0.80 & -0.12 & 0.62 & 0.20 & 1.75 & 0.51 \\
\hline & $50.0 \%$ & 1.95 & 1.70 & -3.09 & -2.54 & 0.38 & -0.62 & 0.06 & 0.71 & 0.49 & 2.03 & 0.60 \\
\hline & $97.5 \%$ & 2.12 & 1.88 & -2.89 & -2.35 & 0.57 & -0.45 & 0.21 & 0.79 & 1.06 & 2.41 & 0.69 \\
\hline \multirow{5}{*}{$\begin{array}{l}\text { Full } \\
\qquad I=10\end{array}$} & Mean & 1.92 & 1.70 & -3.24 & -2.41 & 0.50 & -0.53 & -0.08 & 0.79 & 0.91 & 1.91 & 0.61 \\
\hline & SD & 0.11 & 0.11 & 0.25 & 0.27 & 0.10 & 0.11 & 0.11 & 0.08 & 0.39 & 0.40 & 0.08 \\
\hline & $2.5 \%$ & 1.70 & 1.49 & -3.75 & -3.03 & 0.29 & -0.76 & -0.32 & 0.64 & 0.41 & 1.25 & 0.47 \\
\hline & $50.0 \%$ & 1.92 & 1.69 & -3.24 & -2.38 & 0.50 & -0.53 & -0.08 & 0.79 & 0.80 & 1.85 & 0.61 \\
\hline & $97.5 \%$ & 2.12 & 1.91 & -2.71 & -1.94 & 0.70 & -0.31 & 0.12 & 0.95 & 1.85 & 2.87 & 0.78 \\
\hline \multirow{5}{*}{$\begin{array}{l}\text { Full } \\
\qquad I=60\end{array}$} & Mean & 1.97 & 1.48 & -3.36 & -2.36 & 0.53 & -0.51 & 0.03 & 0.73 & 1.76 & 1.87 & 0.55 \\
\hline & SD & 0.07 & 0.09 & 0.14 & 0.13 & 0.10 & 0.11 & 0.10 & 0.06 & 0.52 & 0.22 & 0.05 \\
\hline & $2.5 \%$ & 1.82 & 1.31 & -3.64 & -2.62 & 0.33 & -0.72 & -0.14 & 0.61 & 0.99 & 1.47 & 0.45 \\
\hline & $50.0 \%$ & 1.97 & 1.48 & -3.35 & -2.36 & 0.53 & -0.51 & 0.03 & 0.73 & 1.67 & 1.86 & 0.54 \\
\hline & $97.5 \%$ & 2.11 & 1.66 & -3.12 & -2.12 & 0.71 & -0.30 & 0.23 & 0.84 & 2.88 & 2.33 & 0.65 \\
\hline \multirow{6}{*}{$\begin{array}{l}\text { Full } \\
\qquad I=400\end{array}$} & Mean & 1.97 & 1.73 & -3.15 & -2.51 & 0.41 & -0.62 & 0.06 & 0.70 & 0.67 & 2.00 & 0.59 \\
\hline & SD & 0.08 & 0.09 & 0.09 & 0.11 & 0.09 & 0.10 & 0.10 & 0.04 & 0.31 & 0.18 & 0.05 \\
\hline & $2.5 \%$ & 1.81 & 1.56 & -3.32 & -2.72 & 0.22 & -0.82 & -0.14 & 0.62 & 0.19 & 1.69 & 0.51 \\
\hline & $50.0 \%$ & 1.97 & 1.73 & -3.15 & -2.51 & 0.41 & -0.61 & 0.07 & 0.70 & 0.64 & 1.99 & 0.59 \\
\hline & $97.5 \%$ & 2.13 & 1.89 & -2.98 & -2.32 & 0.59 & -0.41 & 0.27 & 0.78 & 1.37 & 2.37 & 0.69 \\
\hline & True & 2.00 & 1.50 & -3.00 & -2.50 & 0.50 & -0.50 & 0.00 & 0.70 & 0.80 & 2.00 & 0.50 \\
\hline
\end{tabular}

the occurrence of out-of-stocks (assuming all products were available for every single period and market). In both cases, we used the following weakly informative prior distributions: $\theta \sim N\left(0,100 I_{8}\right), \Sigma_{j j} \sim$ scaled inverse chi-square $(\mathrm{df}=3$; scale $=1)$; and $\sigma_{\xi}^{2} \sim$ scaled inverse chi-square $(\mathrm{df}=-1$; scale $=0.01)$. The results are presented in Table 2 and they are based on a single run of 100,000 iterations from an MCMC sampler, where the last 50,000 iterations are used for parameter estimation.

From the results in Table 2, we observe important differences in terms of parameter inference comparing the case where out-of-stocks are modeled with the one in which out-of-stocks are ignored. Considering the results in the first three blocks of Table 2, it is evident that when out-of-stocks are ignored, our inferences about the model parameters are biased, especially when out-of-stocks are more frequent (see the first and second blocks of Table 2). Specifically, when $I=10$, the $95 \%$ posterior probability intervals for most of the components of $\theta$ do not cover the corresponding true values. For example, $\theta_{z_{1}, x_{3}}$, which is used to derive the mean utility of all the purchase options, is underestimated when out-of-stocks are ignored (the true value is $-3 ; 95 \%$ posterior probability interval, $[-4.60,-3.78])$. In addition, the heterogeneity in the random coefficients for the continuous variable $\left(\Sigma_{x_{4}, x_{4}}\right.$; the true value is $2 ; 95 \%$ posterior probability interval, $[0.54,1.46])$ is underestimated. As expected, the estimation results for the naïve model improve as out-of-stocks become less frequent. In particular, in the third case, where out-of-stocks are on-average observed only in $1.3 \%$ of the time periods for each alternative $(I=400)$, the results are very similar across both models (see the third and sixth blocks in Table 2), a good sign for our approach.

In terms of the full model, the results in the last three blocks in Table 2 show that the method recovers well the original parameters under each of the three scenarios. In fact, the posterior means are close to 
their true values (on average, within 0.8 posterior standard deviations from the corresponding true values). In addition, in all but four cases $\left(\theta_{z_{1}, x_{3}}\right.$ and $\Sigma_{x_{3}, x_{3}}$ in the fifth block and $\theta_{z_{1}, x_{2}}$ and $\Sigma_{\xi}$ in the sixth block of Table 2), the true values are contained within the $95 \%$ posterior probability intervals.

Finally, it is important to mention that we also conducted an additional simulation study by generating 50 data sets for the case where $I=10$. We estimated the model parameters using the proposed method and also ignoring the occurrence of out-of-stocks. The results of this simulation study confirm the basic findings discussed in this subsection (please refer to Online Appendix A, provided in the e-companion). ${ }^{14}$

\subsection{Estimating Lost Sales}

An important factor that determines inventory levels in retailing is the cost of shortage. This cost is closely related to the behavior of customers that encounter an out-of-stock. The cost of an out-of-stock increases with (i) the markup of the product that sells out, and (ii) the fraction of customers that choose not to purchase after experiencing an out-of-stock. ${ }^{15}$ The former is known by the store manager, but the latter is not directly observable. In what follows, we show how to use the model to estimate lost sales-the fraction of customers that chose not to purchase but would have purchased if some of the out-of-stock products had been available - for a given inventory policy. This estimate can be used to compute performance measures such as the fill rate, defined as the fraction of demand filled from stock, which is commonly used in retail operations management. It can also be used together with markup information to assign a dollar value to the cost of shortage.

Consider a customer $i$ facing a set of available products $a_{i}$ who chose the no-purchase option (there is a slight abuse of notation here, because $a_{i}$ is now a set rather than a vector). We drop the time $(t)$ and market $(m)$ subscripts for ease of exposition. The set of out-of-stock products, denoted by $a_{i}^{c}=\mathscr{E} \backslash a_{i}$, includes product $k$. The probability that customer $i$ would have purchased product $k$ had it been available is given by

$\operatorname{Pr}\left(\right.$ choose $k \mid$ choose 0 from $\left.a_{i}\right)$

$$
\begin{aligned}
= & \int \operatorname{Pr}\left(\text { choose } k \mid \text { choose } 0 \text { from } a_{i}, \beta_{i}\right) \\
& \cdot f\left(\beta_{i} \mid \text { choose } 0 \text { from } a_{i}\right) d \beta_{i} \\
= & \int \frac{e^{V_{i k}}}{1+\sum_{\left.j \in a_{i} \cup k\right\}} e^{V_{i j}}} f\left(\beta_{i} \mid \text { choose } 0 \text { from } a_{i}\right) d \beta_{i},
\end{aligned}
$$

\footnotetext{
${ }^{14}$ An electronic companion to this paper is available as part of the online version that can be found at http://mansci.journal.informs.org/.

${ }^{15}$ Also note that the cost of an out-of-stock decreases with the markup of the products that capture the demand for the nonavailable products and leads to the possibility of "strategic out-ofstocks" by the retailer, which we do not pursue here.
}

where $\beta_{i}$ is the (random) preference coefficient of the customer, $f\left(\beta_{i} \mid \cdot\right)$ its conditional density, and $V_{i j} \equiv$ $\beta_{i} x_{j}+\xi_{j}$ is the conditional expected utility of purchasing product $j$. The first equality comes from conditioning on the customer's preferences $\left(\beta_{i}\right)$. The second equality comes from standard properties of the multinomial logit choice model. ${ }^{16}$ Similarly, the probability that the customer would have chosen any of the missing alternatives had they been available is given by

$$
\begin{aligned}
& \operatorname{Pr}\left(\text { choose } a_{i}^{c} \mid \text { choose } 0 \text { from } a_{i}\right) \\
& \quad=\int \frac{\sum_{k \in a_{i}^{c}} e^{V_{i k}}}{1+\sum_{j \in \mathcal{F}} e^{V_{i j}}} f\left(\beta_{i} \mid \text { choose } 0 \text { from } a_{i}\right) d \beta_{i} .
\end{aligned}
$$

There are two challenges in computing lost sales via Equations (13) and (14): (1) the conditional density $f\left(\beta_{i} \mid\right.$ choose 0 from $\left.a_{i}\right)$ is difficult to compute analytically, and (2) the set of available products available to each customer is not always known, the problem considered in this paper. Therefore, it is more convenient to estimate lost sales using simulation, which we describe next.

Our goal is to forecast expected lost sales for a period with initial inventories $I_{1}, \ldots, I_{J}$ generating estimates of lost sales in each of the $R$ iterations of the Gibbs sampler. Specifically, for each iteration $r$, we obtain a draw of the parameters $\left(\theta, \Sigma, \Sigma_{\xi}\right)$ from the MCMC simulation. Then, we generate $N$ new draws of $\beta_{i}$ from a $\operatorname{MVN}\left(\theta^{\prime} Z, \Sigma\right)$ (one for each customer) and $\xi$ from a $\operatorname{MVN}\left(0, \Sigma_{\xi}\right)$. These can be used to calculate the expected utilities $V_{i j}=\beta_{i} x_{j}+\xi_{j}$ for each customer and product.

Next, we sequentially simulate the choices of each customer. Specifically, given the initial inventories, we determine the set of products available to the first customer, $a_{1}$, and sample the customer choice, $y_{1}$, from a multinomial distribution with probabilities specified by (3). Given this choice, we update inventories by subtracting one unit from the chosen product (unless $y_{1}=0$, in which case inventories remain unchanged) and proceed to next customer. The process is repeated for all the $N$ customers. Customers who chose the no-purchase alternative are recorded in the set $O_{r}$ (keeping the corresponding values of the $V_{i j}$ 's and $a_{i}$ ). Accordingly, the expected lost sales can be estimated as follows:

$$
\mathrm{E}(\text { LostSales })=\frac{1}{|R|} \sum_{r=1}^{R} \sum_{i \in O_{r}} \frac{\sum_{k \in a_{i}^{c}} e^{V_{i k}^{(r)}}}{1+\sum_{j \in \mathcal{F}} e^{V_{i j}^{(r)}}},
$$

\footnotetext{
${ }^{16}$ Conditioning on choosing the no-purchase option, the random utility $U_{i 0}$ is distributed according to an extreme value distribution with mode (location) equal to $\ln \left(1+\sum_{j \in a_{i}} e^{V_{i j}}\right)$ and scale equal to 1 . Hence, the probability of preferring $k$ over the no-purchase option is $e^{V_{i k}} \cdot\left(1+e^{V_{i k}}+\sum_{j \in a_{i}} e^{V_{i j}}\right)^{-1}$.
} 
where $V_{i j}^{(r)}$ denotes the value of $V_{i j}$ in iteration $r$. This simulation procedure can be used to forecast lost sales for any initial inventory levels $I_{1}, \ldots, I_{J}$. Alternatively, one could estimate lost sales retrospectively for a time period where the observed data on inventories and sales were collected. The only adjustment to this method is that samples of $\beta_{i}, a_{i}, \xi_{i}$, and $y_{i}$ are obtained directly from the MCMC simulation used in the estimation. This ensures that the distribution of customer preferences and availability are conditioned on the observed data.

We tested the accuracy of this method to estimate expected lost sales using the simulated data described in \$3.5. Actual lost sales were calculated using the simulated utilities by counting customers who chose the outside good, but would have purchased a product under a full assortment. We compared these actual lost sales with those estimated using Equation (15) and the estimated parameters. The correlation between the actual and estimated lost sales is $98.2 \%$, and the mean absolute percentage error is $11.99 \%$. Overall, this method provides a fairly accurate estimate of the expected lost sales for a wide range of inventory levels.

A similar simulation approach can be used to estimate stockout-based substitution. At the end of each iteration $r$, we can store the set of customers $P_{r}$ that purchased any available product. The number of these customers that would have instead purchased the out-of-stock product $k$ when all products are available can be estimated as

$$
\mathrm{E}(\text { Substitution }[k])=\frac{1}{|R|} \sum_{r=1}^{R} \sum_{i \in P_{r}} \frac{e^{V_{i k}^{(r)}}}{1+\sum_{j \in \mathcal{F}} e^{V_{i j}^{(r)}}} .
$$

The next section describes an application of the methodology based on data from the shampoo product category.

\section{Empirical Application: Demand Estimation in the Shampoo Product Category}

We use data on shampoo purchases from six supermarket stores located in different regions of Spain to illustrate the methodology. The stores are owned by a major supermarket chain, with more than 400 stores in this country. The data set was collected from six of these stores including daily sales during 15 consecutive days (excluding two Sundays in which stores are closed) between May 13 and May 29, 1999. The product category includes 24 different stock keeping units (SKUs), but not all SKUs were offered in each store during the study period. In addition to the sales data, information about product availability on the shelf was recorded at the beginning and end of each day. In total, 291 days with zero final inventory were recorded across all days, stores, and products.

The data also contain price and promotion information for every product on each store-day. From the 24 products in the data set, 16 products exhibited price variation across stores, but very few products exhibited temporal price variation within a given store during the study period. Table 3 displays the brands of each product and shows summary statistics for each of the products in terms of daily unit sales, prices (in Spanish pesetas), promotion incidence, availability, and number of stores where each product is offered. ${ }^{17}$

\subsection{Model Specification}

We include nine covariates $\left(X_{j t m}\right)$ in the utility function of each customer. Two of these covariates have random coefficients: Price and Purchase option. The latter is a dummy variable equal to 1 in every period for all options except the no-purchase option. This variable captures total category demand for shampoo products as higher coefficients associated to this variable raise the utility of all purchase alternatives in relation to the utility of the no-purchase option. In addition, we include dummy variables for the following brands: Pantene, Herbal Essences, Head \& Shoulders (H\&S), Cabello Sano, and Timotei. We also include a dummy variable (Weekend) to control for seasonality (changes in demand during weekends). This variable is equal to 1 for all purchase alternatives for every time period corresponding to a Saturday and is equal to 0 otherwise (recall that the stores are closed on Sundays). Finally, we control for promotional effects by using a dummy variable (Promotion) equal to 1 if an item was promoted and zero otherwise during a given time period and store. We note that these last two variables (Weekend and Promotion) also enable us to explicitly consider in the utility function changes in demand that might be anticipated by the store manager, given that promotional activity can (and is expected) to cause increased demand.

We use store-level demographic information to capture observed preference heterogeneity across stores (unobserved heterogeneity is captured through random coefficients). Specifically, we collected information on average declared income for each market. Accordingly, the vector of demographic variables for a given store $m\left(Z_{m}\right)$ has two components: an indicator equal to 1 for all stores (intercept) and the standardized natural logarithm of income. ${ }^{18}$ In addition,

\footnotetext{
${ }^{17}$ Availability is measured as the fraction of days with zero end inventory for a given product.

${ }^{18}$ We also experimented using additional demographic information (e.g., age), but the results and the fit of the model did not substantially change.
} 
Table 3 Brands and Summary Statistics for the Shampoo Data: Brands, Means, and Standard Deviations of Daily Sales and Prices, and Promotion Incidence, Fraction of Out-of-Stocks (00S), and Number of Stores for Each Product

\begin{tabular}{|c|c|c|c|c|c|c|c|c|}
\hline \multirow[b]{2}{*}{ Product } & \multirow[b]{2}{*}{ Brand } & \multicolumn{2}{|c|}{ Daily unit sales } & \multicolumn{2}{|c|}{ Price } & \multirow{2}{*}{$\begin{array}{l}\text { Promotion } \\
\text { incidence }\end{array}$} & \multirow{2}{*}{$\begin{array}{c}\text { OOS } \\
\text { incidence }\end{array}$} & \multirow{2}{*}{$\begin{array}{c}\text { Number of } \\
\text { stores }\end{array}$} \\
\hline & & Mean & SD & Mean & SD & & & \\
\hline 1 & Other & 0.08 & 0.27 & 3.99 & 0.00 & 0.00 & 0.00 & 6 \\
\hline 2 & Other & 0.00 & 0.00 & 4.97 & 0.04 & 0.00 & 0.11 & 3 \\
\hline 3 & Other & 0.07 & 0.25 & 4.97 & 0.04 & 0.00 & 0.12 & 4 \\
\hline 4 & Timotei & 0.12 & 0.39 & 6.03 & 0.10 & 0.00 & 0.27 & 6 \\
\hline 5 & Timotei & 0.12 & 0.33 & 5.99 & 0.27 & 0.00 & 0.10 & 6 \\
\hline 6 & Timotei & 0.17 & 0.43 & 6.03 & 0.10 & 0.00 & 0.26 & 6 \\
\hline 7 & Timotei & 0.06 & 0.23 & 5.79 & 0.41 & 0.00 & 0.16 & 5 \\
\hline 8 & Other & 0.03 & 0.18 & 4.72 & 0.32 & 0.00 & 0.38 & 4 \\
\hline 9 & Other & 0.06 & 0.23 & 4.75 & 0.00 & 0.00 & 0.20 & 6 \\
\hline 10 & Pantene & 0.17 & 0.37 & 4.50 & 0.11 & 0.20 & 0.33 & 6 \\
\hline 11 & Pantene & 0.28 & 0.97 & 4.50 & 0.16 & 0.20 & 0.40 & 5 \\
\hline 12 & Pantene & 0.29 & 0.89 & 4.50 & 0.11 & 0.18 & 0.39 & 6 \\
\hline 13 & Pantene & 0.28 & 0.73 & 4.45 & 0.15 & 0.20 & 0.39 & 6 \\
\hline 14 & Other & 0.07 & 0.29 & 4.99 & 0.00 & 0.00 & 0.17 & 6 \\
\hline 15 & Other & 0.24 & 0.53 & 2.07 & 0.03 & 0.00 & 0.11 & 5 \\
\hline 16 & Other & 0.24 & 0.53 & 4.85 & 0.00 & 0.00 & 0.03 & 6 \\
\hline 17 & Herbal Essences & 1.03 & 1.47 & 4.55 & 0.19 & 0.63 & 0.08 & 6 \\
\hline 18 & $\mathrm{H} \& \mathrm{~S}$ & 0.32 & 0.68 & 5.20 & 0.22 & 0.00 & 0.36 & 6 \\
\hline 19 & $\mathrm{H} \& \mathrm{~S}$ & 0.21 & 0.63 & 5.17 & 0.19 & 0.00 & 0.31 & 6 \\
\hline 20 & Other & 0.19 & 0.45 & 4.99 & 0.00 & 0.00 & 0.11 & 6 \\
\hline 21 & Cabello Sano & 0.11 & 0.32 & 3.45 & 0.00 & 0.01 & 0.12 & 5 \\
\hline 22 & Cabello Sano & 0.27 & 0.61 & 3.45 & 0.00 & 0.01 & 0.08 & 6 \\
\hline 23 & Cabello Sano & 0.30 & 0.64 & 3.47 & 0.04 & 0.00 & 0.42 & 6 \\
\hline 24 & Other & 0.06 & 0.23 & 4.25 & 0.00 & 0.00 & 0.17 & 5 \\
\hline
\end{tabular}

the size of the market for each store was estimated combining data on population and total consumption of shampoo in Spain. ${ }^{19}$ We note that, as is common in the marketing and economics literature, the market size is assumed to be constant across time periods, although it is important to highlight that our model allows for a different fraction of the market to make purchases in different periods. ${ }^{20}$

\subsection{Results}

Using the method introduced in $\S 3$, we estimated the model based on the covariates and demographic variables described previously. We also estimated a benchmark model that ignores out-of-stocks, i.e., every product offered in a given store is assumed to be available to all customers in every time period.

\footnotetext{
${ }^{19}$ The population data were downloaded from http://www.ine.es, whereas the consumption data were obtained from Euromonitor International. We also experimented with an alternative definition of the market size (approximately twice the size of the one used in this section) and obtained very similar results.

${ }^{20}$ We also tested the consequences of this assumption using simulated data where the market size fluctuates over time and estimating this model assuming the market size is constant and equal to its average. When the variability of the market size increases, we find a slight decrease in the estimated variance of the intercepts that affect the utility of all purchase alternatives, and a slight increase in the estimated variance of the demand shocks. The mean of consumer coefficients does not exhibit any systematic changes. Detailed results are available from the authors upon request.
}

However, products that were never available in a particular store were excluded from the choice set in the benchmark model. We computed the log-marginal likelihood for the full and benchmark models and obtained a log-Bayes factor equal to 74.5, which gives very strong empirical support for the full model (Kass and Raftery 1995). Table 4 reports the estimation results for the hyperparameters $\theta, \Sigma$, and $\sigma_{\xi}^{2}$ under both model specifications.

From the results of the full model, we note that products from the brands Herbal Essences and H\&S are on average more demanded than all other products (see the results for $\theta_{\text {Intercept }}$ in Table 4). Furthermore, the results for $\theta_{\text {Income }}$ show higher intrinsic demand for Pantene and H\&S products in stores reaching customers with higher income levels. As expected, the price, weekend, and promotion effects (see $\theta_{\text {Intercept, Price, }} \theta_{\text {Intercept, Weekend }}$, and $\theta_{\text {Intercept, Promotion }}$ ) show a significant disutility associated with higher prices and higher levels of demand during Saturdays and when items are promoted. These results also show that the promotional and weekend effects are stronger for lower-income markets.

In addition to the observed heterogeneity across stores captured by $\theta_{\text {Income }}$, the results for $\Sigma$ show the magnitude of the unobserved heterogeneity within stores in terms of total demand (Purchase option) and price sensitivity (Price). The variances for these random coefficients are estimated as 0.70 and 0.24 , 
Empirical Results: Estimated Posterior Means, Standard Deviations, and $25 \%$ and $75 \%$ quantiles for $\theta, \Sigma$, and $\sigma_{\xi}$ (Iterations 200,001-400,000)

\begin{tabular}{|c|c|c|c|c|c|c|c|c|c|}
\hline & & \multicolumn{4}{|c|}{ Full model } & \multicolumn{4}{|c|}{ Ignoring out-of-stocks } \\
\hline \multicolumn{2}{|c|}{ Variable } & Mean & SD & $25 \%$ & $75 \%$ & Mean & SD & $25 \%$ & $75 \%$ \\
\hline \multirow{9}{*}{$\begin{array}{r}\text { Main effects } \\
\left(\theta_{\text {Intercept, }}\right)\end{array}$} & Pantene & 0.78 & 0.23 & 0.62 & 0.94 & 0.45 & 0.22 & 0.30 & 0.59 \\
\hline & Herbal Essences & 1.34 & 0.34 & 1.11 & 1.58 & 1.22 & 0.30 & 1.01 & 1.43 \\
\hline & $H \& S$ & 1.31 & 0.27 & 1.13 & 1.49 & 1.08 & 0.24 & 0.92 & 1.25 \\
\hline & Cabello Sano & 0.68 & 0.23 & 0.52 & 0.84 & 0.48 & 0.24 & 0.32 & 0.65 \\
\hline & Timotei & 0.43 & 0.31 & 0.22 & 0.64 & 0.30 & 0.31 & 0.10 & 0.51 \\
\hline & Promotion & 0.94 & 0.29 & 0.75 & 1.14 & 1.21 & 0.27 & 1.02 & 1.39 \\
\hline & Weekend & 1.06 & 0.22 & 0.91 & 1.20 & 1.16 & 0.24 & 0.99 & 1.32 \\
\hline & Purchase option & -5.10 & 0.57 & -5.46 & -4.67 & -5.06 & 0.56 & -5.42 & -4.69 \\
\hline & Price & -0.85 & 0.19 & -0.97 & -0.72 & -0.95 & 0.20 & -1.08 & -0.81 \\
\hline \multirow{9}{*}{$\begin{array}{l}\text { Income } \\
\text { interactions } \\
\left(\theta_{\text {Income },}\right)\end{array}$} & Pantene & 0.24 & 0.23 & 0.08 & 0.39 & 0.29 & 0.23 & 0.14 & 0.44 \\
\hline & Herbal Essences & -0.15 & 0.34 & -0.37 & 0.09 & -0.10 & 0.33 & -0.33 & 0.13 \\
\hline & $H \& S$ & 0.57 & 0.26 & 0.40 & 0.74 & 0.66 & 0.25 & 0.48 & 0.83 \\
\hline & Cabello Sano & 0.09 & 0.24 & -0.08 & 0.25 & -0.08 & 0.23 & -0.23 & 0.07 \\
\hline & Timotei & -0.01 & 0.32 & -0.21 & 0.21 & 0.17 & 0.31 & -0.04 & 0.38 \\
\hline & Promotion & -0.43 & 0.29 & -0.62 & -0.23 & -0.51 & 0.29 & -0.71 & -0.32 \\
\hline & Weekend & -0.23 & 0.21 & -0.37 & -0.09 & -0.22 & 0.22 & -0.36 & -0.08 \\
\hline & Purchase option & -0.38 & 0.53 & -0.73 & -0.02 & -0.37 & 0.52 & -0.70 & -0.03 \\
\hline & Price & -0.05 & 0.12 & -0.13 & 0.03 & -0.07 & 0.12 & -0.15 & 0.02 \\
\hline \multirow{2}{*}{$\begin{array}{l}\text { Heterogeneity } \\
\qquad(\Sigma)\end{array}$} & Purchase option & 0.70 & 0.39 & 0.42 & 0.89 & 0.86 & 0.80 & 0.42 & 0.94 \\
\hline & Price & 0.24 & 0.09 & 0.17 & 0.28 & 0.29 & 0.12 & 0.20 & 0.35 \\
\hline \multirow{2}{*}{\multicolumn{2}{|c|}{ Demand shock }} & & & & & & & & \\
\hline & & 1.00 & 0.04 & 0.97 & 1.03 & 1.00 & 0.04 & 0.98 & 1.03 \\
\hline
\end{tabular}

respectively. The results for the variance of the demand shocks $\left(\sigma_{\xi}^{2}\right)$ suggest that these unobserved demand effects are substantial (the posterior mean of $\sigma_{\xi}^{2}$ is estimated as 1.00).

Finally, when comparing the full and benchmark models, we note that the posterior means of $\theta_{\text {Intercept }}$ for the brands Pantene, H\&S, and Cabello Sano are smaller under the benchmark model than in the full model (these posterior means are approximately one posterior standard deviation from each other). These differences do not exhibit a strong level of statistical significance, but their direction suggests that ignoring out-of-stocks leads to lower estimates of demand for these brands. In contrast, the corresponding posterior mean for Herbal Essences is very similar across both models, which is consistent with the high availability of this product on the shelf $(92 \%$, see fraction of outof-stocks for product 17 in Table 3).

We tested the robustness of these results with respect to a potential inventory endogeneity bias. Using the extensions described in \$3.4, we accounted for a possible correlation between inventory and the demand shocks by adding Equation (12) into the estimation, which helps to mitigate this endogeneity problem. We used the products' cost markup as an instrumental variable, which varies across products and stores but not across days. The results from this extended model suggest a small correlation between inventory and demand shocks (the estimated posterior mean of the correlation between $\nu$ and $\xi$ is equal to 0.26)..$^{21}$ Furthermore, there is strong empirical support favoring the model where the correlation between $\xi$ and $\nu$ is zero (log-Bayes factor $=-716.6$ ). The estimated marginal posterior distribution of each parameter in the extended model exhibits a reasonable precision, suggesting that the parameters are identified after controlling for inventory endogeneity (see Allenby and Rossi 2003 for more details on identification of Bayesian IV models). In addition, the estimated demand parameters from this extended model and the full model presented in $\$ 4.2$ are very similar (results are available from the authors upon request), which implies that the potential bias due to inventory endogeneity is small. Recall that the demand specification of this application includes factors that measure seasonality, promotional activity, and brand effects, capturing most of the variation in demand that can be anticipated by the store manager. This helps to reduce endogeneity problems arising from omitted variables in the demand specification.

\section{Estimating and Mitigating the Costs of Out-of-Stocks}

Using the expressions derived in $\$ 3.6$ (see Equation (15)), we estimated lost sales for every time

\footnotetext{
${ }^{21}$ Interestingly, we obtained a positive coefficient for price margin, which is consistent with theoretical predictions from the inventory management literature (e.g., Porteus 2002). Detailed results about this sensitivity analysis are available from the authors upon request.
} 
Figure 1 Percentage of Lost Purchases as a Fraction of Full-Availability Sales vs. Number of Out-of-Stock Products

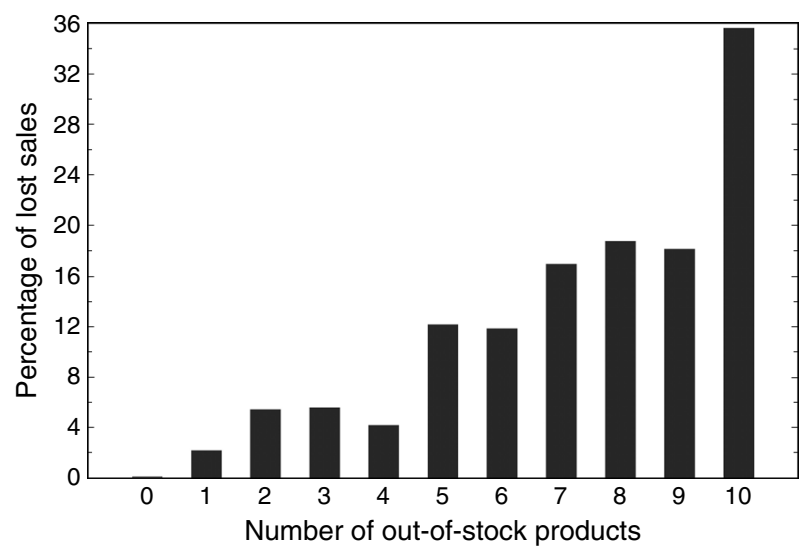

period in each store. We are particularly interested in estimating how the number of out-of-stock products affects lost sales. In our application, the number of products simultaneously out of stock on a given day ranges between 0 and 10 SKUs. Our methodology is sufficiently flexible to estimate lost sales for all of these possible scenarios without increasing the computational complexity of the method. Figure 1 shows the estimated average lost sales as a function of the number of out-of-stock products. The figure reveals that lost sales increases nonlinearly in the number of products not available. When five or fewer products are out of stock the average lost sales are equal to $5.9 \%$ of the full-availability sales. But when six or more products are out of stock, lost sales grow by more than three times, up to $20.2 \%$ of the fullavailability sales. This suggests that the first products that stock out have a smaller impact on category sales, but lost sales increase more rapidly as more products become unavailable. This pattern has important implications for assortment planning and inventory replenishment decisions. In supermarkets, there are substantial fixed costs of replenishing products on the shelf. Hence, Figure 1 suggests that replenishments may be postponable until several products become out of stock without substantially affecting category sales, although the profitability consequences of such a policy will depend on the retail margins of each of the products and the stockout-based substitution patterns.

Note that in this analysis lost sales are estimated for each period and store in our data set. Alternatively, one could estimate lost sales for a new time period by simulating a sequence of consumers making choice decisions under limited and infinite inventory levels. In the context of the MCMC estimation, this analysis can be easily performed, and we use these results for a particular store (store 5) to illustrate how consumers switch from one product to another when their most preferred item is not available. As we show in Table 5, for the segment of customers who intend to buy each product $i$ (rows), we estimate the percentage that choose instead alternative $j$ (columns) considering representative levels of product inventory and the utility function covariates. The NP column shows the customer that choose not to purchase, which are counted as lost sales. This analysis enables us to provide a detailed characterization of the expected consequences of out-of-stocks on customers' buying behavior. ${ }^{22}$

Specifically, the diagonal elements in the first block of this matrix indicate the percentage of customers that are able to find their most preferred product and, consequently, provide an estimate of the level of "service" provided by the retailer to its customers. Considering all alternatives (not just those reported in Table 5), although some products exhibit very high levels of availability (e.g., products 5, 6, 17, and 22), most of them show availability levels below 50\% (e.g., product 10). In this regard, it is informative to consider the off-diagonal elements of this switching matrix. For example, when product 10 (Pantene) is not available, most customers intending to buy that item would not buy any of the available products: $37.3 \%$ of the customers intending to buy product 10 choose the no-purchase option compared to only $13.7 \%$ (i.e., $100 \%-49.0 \%-37.3 \%$ ) that substitute this product with an available one. Among the latter group of customers, most of these stockout-based substitutions correspond to purchases of product 17 (Herbal Essences). Overall, we estimate that for most products, between one- and two-thirds of the customers that intend to buy a product are not able to find their most preferred alternative and decide not to buy. Finally, the last column of Table 5 shows the change in contribution margin due to lost sales and stockout-based substitution. For most products with low availability ( $20 \%$ or below), contribution margins decrease between $30 \%$ and $65 \%$. Overall, this analysis suggests that the financial consequences of out-ofstocks can be sizable.

In addition to estimating stockout-based substitution and lost sales, we also evaluate the effect of a strategy that seeks to mitigate the effect of out-ofstocks: conducting a temporary price reduction for a single product to recapture a fraction of lost sales. Price discounts increase the attractiveness of substitute products, inducing some customers whose preferred product is not available to substitute their intended purchase with another product rather than choose the no-purchase option. In what follows, we

\footnotetext{
${ }^{22}$ For space considerations, we display the results of this analysis for only 10 of the 24 products for store 5 . Therefore, the sum of the substitution rates in each of the rows of Table 5 is not equal to 1 .
} 
Estimated Switching Matrix, Full-Availability Sales, and Change in Contribution Margin for Selected Products

\begin{tabular}{|c|c|c|c|c|c|c|c|c|c|c|c|c|c|c|}
\hline \multirow{3}{*}{$\begin{array}{l}\text { Intended } \\
\text { choice }\end{array}$} & \multirow[b]{3}{*}{ Brand } & \multicolumn{11}{|c|}{ Actual choice } & \multirow{3}{*}{$\begin{array}{c}\text { Expected } \\
\text { demand } \\
\text { (units) }\end{array}$} & \multirow{3}{*}{$\begin{array}{l}\text { Contribution } \\
\text { margin } \\
\text { reduction (\%) }\end{array}$} \\
\hline & & 4 & 5 & 6 & 8 & 10 & 17 & 18 & 19 & 22 & 23 & 25 & & \\
\hline & & $\mathrm{T}$ & $\mathrm{T}$ & $\mathrm{T}$ & 0 & $P$ & $\mathrm{HE}$ & $H \& S$ & $H \& S$ & CS & CS & NP & & \\
\hline 4 & $\mathrm{~T}$ & 20.1 & 3.9 & 3.6 & 0.0 & 0.0 & 17.2 & 0.0 & 0.0 & 2.5 & 0.0 & 44.5 & 2.2 & 53.0 \\
\hline 5 & $\mathrm{~T}$ & 0.0 & 100.0 & 0.0 & 0.0 & 0.0 & 0.0 & 0.0 & 0.0 & 0.0 & 0.0 & 0.0 & 2.1 & 0.0 \\
\hline 6 & $T$ & 0.0 & 0.0 & 99.8 & 0.0 & 0.0 & 0.0 & 0.0 & 0.0 & 0.0 & 0.0 & 0.1 & 2.1 & 0.2 \\
\hline 8 & 0 & 0.2 & 2.6 & 2.7 & 5.5 & 0.8 & 13.7 & 0.3 & 0.4 & 1.8 & 0.1 & 63.2 & 1.6 & 64.4 \\
\hline 10 & $P$ & 0.0 & 1.0 & 1.3 & 0.0 & 49.0 & 6.8 & 0.0 & 0.0 & 1.3 & 0.0 & 37.3 & 4.7 & 32.0 \\
\hline 17 & $\mathrm{HE}$ & 0.0 & 0.0 & 0.0 & 0.0 & 0.0 & 99.9 & 0.0 & 0.0 & 0.0 & 0.0 & 0.1 & 19.2 & 0.1 \\
\hline 18 & $\mathrm{H} \& \mathrm{~S}$ & 0.0 & 2.4 & 2.7 & 0.0 & 0.0 & 14.0 & 20.4 & 0.0 & 1.9 & 0.0 & 52.3 & 3.6 & 46.9 \\
\hline 19 & $\mathrm{H} \& \mathrm{~S}$ & 0.0 & 2.4 & 2.5 & 0.0 & 0.0 & 13.7 & 0.0 & 19.4 & 2.1 & 0.0 & 53.3 & 3.4 & 48.0 \\
\hline 22 & CS & 0.0 & 0.0 & 0.0 & 0.0 & 0.0 & 0.0 & 0.0 & 0.0 & 99.7 & 0.0 & 0.2 & 3.9 & 0.2 \\
\hline 23 & CS & 0.0 & 1.2 & 1.2 & 0.0 & 0.0 & 8.9 & 0.0 & 0.0 & 1.7 & 20.9 & 61.6 & 4.0 & 55.9 \\
\hline
\end{tabular}

Notes. Within the first block of this table, cell entries $(i, j)$ where $i$ indexes row and $j$ indexes column indicate the percentage that choose alternative $j$ among the segment of customers who intend to buy product i. 0, Other; T, Timotei; P, Pantene; HE, Herbal Essences; CS, Cabello Sano; NP, no purchase. Expected demand indicates the total number of units that would be sold during 15 days if all products were available. Contribution margin reduction indicates the percentage of forgone contribution margin as a fraction of the total contribution margin that would be obtained if all products were available.

show how to use our model to quantify the expected fraction of lost sales that would be recaptured (from the no-purchase option) through these temporary price promotions. Consider the set $O_{A}$ of customers who chose not to purchase when assortment $A$ was available. Suppose that the price of product $k \in A$ is discounted by some fraction of the original price. For each customer $i \in O_{A}$, we define two events: (1) $E_{i 1}$ is the event that customer $i$ would have purchased some of the unavailable products (i.e., products in $A^{c}$ ) had they been available; (2) $E_{i 2}$ is the event that the customer would purchase the discounted product $k$ when only products in $A$ are available. All customers in $O_{A}$ experiencing $E_{i 1}$ are counted as lost sales; those who in addition experience $E_{i 2}$ count as recaptured lost sales. Therefore, the fraction of lost sales that is recaptured can be calculated as

$$
\text { Lost Sales Reduction }=\frac{\sum_{i \in O_{A}} \operatorname{Pr}\left(E_{i 1}, E_{i 2} \mid i \in O_{A}\right)}{\text { LostSales }},
$$

where LostSales can be estimated using the simulation procedure described in \$3.6. Details on how to calculate the numerator of the expression above are shown in Online Appendix B, provided in the e-companion.

Accordingly, the model was used to measure the effectiveness of implementing price reductions of $20 \%$ for different store-days. We consider scenarios where only one product is discounted at a time. As an illustration, we report results for two store-days with very different levels of availability: (i) day 3 in market 5, where 10 SKUs have zero final inventory; and (ii) day 15 in market 2, where only one product has zero final inventory-SKU 15 (Pantene). In the case of day 3 in market 5 , a price promotion on product 17
(Herbal Essences) reduces lost sales by $2.0 \%$; all other promotions reduce lost sales by less than $1 \%$. However, in the case of day 15 in market 2, it is more effective to discount product 13 (Pantene), which leads to a lost sales reduction of $4.2 \%$. In this case, a promotion of product 17 leads to a $1.6 \%$ reduction in lost sales.

The comparison of the results from these two storedays provides insights on the effectiveness of price promotions at mitigating the consequences of out-ofstocks. In the case of day 15 in market 2, where only one product was out of stock, a price promotion on a product with similar characteristics to the missing product is more effective to recapture lost sales. In contrast, when many products are missing, it is more effective to use a price promotion on a popular product (SKU 17).

These results show that price promotions can be useful to reduce lost sales and improve customer service. However, promotions may also have negative consequences, including (1) a reduction in the margin of the discounted product, and (2) cannibalization of sales from other products with higher margins. ${ }^{23}$ These consequences can also be easily estimated with our model if data on the cost of goods sold by SKU are available. Using gross margin data from the supermarket chain under study, we evaluated the net change in category profits accounting for the effect of increased sales, reduced markups, and cannibalization. In the case of day 3 in market 5, the promotion on product 17 increased total profits by $13.2 \%$.

${ }^{23}$ Other negative effects not considered here include stockpiling effects that occur when customers buy the promoted product earlier because of the discount, which leads to a cannibalization of future sales. 
In contrast, in the case of day 15 in market 2, the price promotion on products 13 and 17 reduces profits by $32.5 \%$ and $10.7 \%$, respectively. ${ }^{24}$

In summary, this section illustrates how the methodology can be used to assess the consequences of policies aimed at mitigating the costs of out-ofstocks (e.g., lost sales reduction, impact on category profits). Many additional policy experiments could be also performed, including discounts on multiple products and experimenting with different magnitudes for price discounts. These additional policy experiments can be easily implemented using the estimated model. Note, however, that our estimation and subsequent analysis do not consider any long-run effects on customer behavior. For example, some of the customers that do not find their most preferred item available might return to the store in future periods to buy the missing product. Alternatively, it is possible that some of these customers might reduce their likelihood of returning to the store in future periods, transferring not only their shampoo purchases, but also those in other product categories, to competing retailers. Studying these long-term effects constitutes a very interesting avenue for future research that would probably require getting access to customer panel data with a longer time series of sales and inventory data from multiple product categories.

\section{Conclusions}

In this paper, we have proposed a method to capture the effects of out-of-stocks on customer behavior using data commonly available to a store manager. Lack of precise availability data in real time, which is common in practical retail settings, introduces a major challenge in identifying the effect of out-of-stocks on sales. To overcome these difficulties, our methodology simulates the transition of the inventory on the shelf conditioning on snapshots of information about product inventory, which can be obtained through a periodic inventory review system. These data are combined with daily sales and pricing data to estimate a structural model of demand for a product category.

The method has several attractive features. First, the model can be estimated using data from multiple stores and markets. Second, our method is applicable in categories with many products and with slowmoving products exhibiting frequent out-of-stocks. Previous methods in this area were limited by the number of products, the type of substitutions, and the number of products that could be simultaneously out of stock. Third, our structural demand model allows

\footnotetext{
${ }^{24}$ Promotions on other products, such as SKU 10, reduced lost sales by about $1 \%$ without substantially changing profits.
}

the evaluation of policies to mitigate the consequences of out-of-stocks (such as temporary price promotions). Fourth, the methodology explicitly models the distribution of the timing of out-of-stocks (which are unobservable in periodic inventory systems), providing useful information on product availability.

In terms of future research, several interesting generalizations can be identified. Considering the demand model, alternative specifications or behavioral assumptions could be studied (e.g., probit model, complexity of choice decisions). In addition, the model could be extended to explicitly consider the possibility of some customers returning to the store in future periods if they did not find a product in a given time period. Because our methodology is based on estimating the joint distribution of availability and sales, another possible extension could be developed for the detection of out-of-stocks based on point-of-sales data. Finally, the model could be generalized to consider purchase quantity decisions (e.g., Kim et al. 2002).

In summary, we hope that the methodology presented in this paper may provide useful tools for researchers and managers to estimate the consequences of out-of-stocks and assess the impact of policies to mitigate their costs. This issue is certainly relevant for audiences in the operations management, marketing, and economics disciplines, and we hope this work stimulates more cross-disciplinary research in this area.

\section{Electronic Companion}

An electronic companion to this paper is available as part of the online version that can be found at http:// mansci.journal.informs.org/.

\section{Acknowledgments}

The authors thank seminar participants at the University of Chicago, University of Michigan, Emory University, University of Rochester, University of Miami, University of Southern California, University of Wisconsin, the 2008 Marketing Science Conference hosted by the University of British Columbia, and the 2008 Workshop on Empirical Research in Operations Management hosted by the Wharton School of the University of Pennsylvania. In particular, they thank J. P. Dubé, Vishal Gaur, Günter Hitsch, Puneet Manchanda, Peter Rossi, S. Sriram, and Naufel Vilcassim for providing very detailed and helpful comments about this paper. They also extend their gratitude to the Jay H. Baker Retailing Initiative at the Wharton School of the University of Pennsylvania and the retail research center CERET at the Industrial Engineering Department of the University of Chile. They thank J. P. Brackman, Ana Gonzalez, and Jim Flannery of Procter \& Gamble for giving them access to the data used in this paper. They also thank the editor, Wallace Hopp, the area editor, and two anonymous reviewers for their helpful suggestions that greatly improved this paper. 


\section{References}

Allenby, G. M., P. E. Rossi. 2003. Bayesian statistics and marketing. Marketing Sci. 22(3) 304-328.

Anderson, E., G. Fitzsimons, D. Simester. 2006. Measuring and mitigating the cost of stockouts. Management Sci. 52(11) 1751-1763.

Andrews, R. L., T. C. Srinivasan. 1995. Studying consideration effects in empirical choice models using scanner panel data. J. Marketing Res. 32(1) 30-41.

Anupindi, R., M. Dada, S. Gupta. 1998. Estimation of consumer demand with stock-out based substitution: An application to vending machine products. Marketing Sci. 17(4) 406-423.

Berry, S. 1994. Estimating discrete choice models of product differentiation. RAND J. Econom. 25(2) 242-262.

Berry, S., J. Levinson, A. Pakes. 1995. Automobile prices in market equilibrium. Econometrica 963(4) 841-890.

Besanko, D., J.-P. Dubé, S. Gupta. 2003. Competitive price discrimination strategies in a vertical channel using aggregate data. Management Sci. 49(9) 1121-1138.

Bruno, H., N. Vilcassim. 2008. Structural demand estimation with varying product availability. Marketing Sci. 27(6) 1126-1131.

Cachon, G. P., M. Olivares. 2010. Drivers of finished-goods inventory in the U.S. automobile industry. Management Sci. 56(1) 202-216.

Cachon, G. P., C. Terwiesch. 2005. Matching Supply with Demand, 1st ed. McGraw-Hill, New York.

Campo, K., E. Gijsbrechts, P. Nisol. 2000. Towards understanding consumer response to stock-outs. J. Retailing 76(2) 219-242.

Campo, K., E. Gijsbrechts, P. Nisol. 2003. The impact of retailer stockouts on whether, how much, and what to buy. Internat. J. Res. Marketing 20(3) 273-286.

Chen, Y., S. Yang. 2007. Estimating disaggregate models using aggregate data via augmentation of individual choice. J. Marketing Res. 44(4) 596-613.

Chintagunta, P. K., J.-P. Dubé. 2005. Estimating a stockkeeping-unitlevel brand choice model that combines household panel data and store data. J. Marketing Res. 42(3) 368-379.

Chintagunta, P. K., D. Jain, N. J. Vilcassim. 1991. Investigating heterogeneity in brand preferences in logit models for panel data. J. Marketing Res. 28(4) 417-428.

Conlon, C., J. H. Mortimer. 2009. Demand estimation under incomplete product availability. Working paper, Harvard University, Cambridge, MA.

DeHoratius, N., A. Raman. 2008. Inventory record inaccuracy: An empirical analysis. Management Sci. 54(4) 627-641.

Fader, P., B. Hardie. 1996. Modeling consumer choice among SKUs. J. Marketing Res. 33(4) 442-452.

Fitzsimons, G. J. 2000. Consumer response to stockouts. J. Consumer Res. 27(2) 249-266.

Gruen, T. W., D. S. Corsten, S. Bharadwaj. 2002. Retail Out-ofStocks: A Worldwide Examination of Extent, Causes and Consumer Responses. Grocery Manufacturers of America, Washington, DC.
Hauser, J., B. Wernerfelt. 1990. An evaluation cost model of consideration sets. J. Consumer Res. 16(4) 393-408.

Jiang, R., P. Manchanda, P. Rossi. 2009. Bayesian analysis of random coefficient logit models using aggregate data. J. Econometrics 149(2) 136-148.

Kalyanam, K., S. Borle, P. Boatwright. 2007. Deconstructing each item's category contribution. Marketing Sci. 26(3) 327-341.

Kass, R. E., A. E. Raftery. 1995. Bayes factors. J. Amer. Statist. Assoc. 90(430) 773-795.

Kim, J., G. M. Allenby, P. E. Rossi. 2002. Modeling consumer demand for variety. Marketing Sci. 21(3) 229-250.

Kök, A. G., M. L. Fisher. 2007. Demand estimation and assortment optimization under substitution: Methodology and application. Oper. Res. 55(6) 1001-1021.

Manchanda, P., P. E. Rossi, P. K. Chintagunta. 2004. Response modeling with nonrandom marketing-mix variables. J. Marketing Res. 41(4) 467-478.

Musalem, A., E. T. Bradlow, J. S. Raju. 2008. Who's got the coupon? Estimating consumer preferences and coupon usage from aggregate information. J. Marketing Res. 45(6) 715-730.

Musalem, A., E. T. Bradlow, J. S. Raju. 2009. Bayesian estimation of random-coefficients choice models using aggregate data. J. Appl. Econometrics 24(3) 490-516.

O'Connell, V. 2008. Reversing field, Macy's goes local. Wall Street Journal (April 21) B1.

Porteus, E. L. 2002. Foundations of Stochastic Inventory Theory. Stanford University Press, Stanford, CA.

Roberts, J. H., J. M. Lattin. 1991. Development and testing of a model of consideration set composition. J. Marketing Res. 28(4) 429-440.

Rossi, P. E., R. McCulloch, G. M. Allenby. 1996. The value of purchase history data in target marketing. Marketing Sci. 15(4) 321-340.

Smith, S. A., N. Agrawal. 2000. Management of multi-item retail inventory systems with demand substitution. Oper. Res. 48(1) 50-64.

Swait, J., T. Erdem. 2002. Sales promotions consistency and consumer preferences. J. Marketing Res. 34(3) 304-320.

Train, K. E. 2003. Discrete Choice Methods with Simulation. Cambridge University Press, Cambridge, MA.

Villas-Boas, J. M., R. S. Winer. 1999. Endogeneity in brand choice models. Management Sci. 45(10) 1324-1338.

Vulcano, G., G. van Ryzin, R. Ratliff. 2008. Estimating primary demand for substitutable products from sales transaction data. Working paper, Stern School of Business, New York University, New York.

Yang, S., Y. Chen, G. M. Allenby. 2003. Bayesian analysis of simultaneous demand and supply. Quant. Marketing Econom. 1(3) 251-275.

Zipkin, P. 2000. Foundations of Inventory Management. McGraw-Hill, New York. 